The Astrophysical Journal, 239:495-501, 1980 July 15

c) 1980. The American Astronomical Society. All rights reserved. Printed in U.S.A.

\title{
LUMINOSITIES AND TEMPERATURES OF THE REDDEST STARS IN THREE LMC CLUSTERS
}

\author{
JAY A. Frogel \\ Cerro Tololo Inter-American Observatory ${ }^{1}$ \\ S. E. Persson \\ Mount Wilson and Las Campanas Observatories, Carnegie Institution of Washington \\ AND \\ Judith G. COHEN \\ Palomar Observatory, California Institute of Technology \\ Received 1979 August 28; accepted 1980 January 22
}

\begin{abstract}
Infrared observations in the 1.2-2.2 $\mu \mathrm{m}$ region are presented for 12 of the reddest stars in the Large Magellanic Cloud (LMC) clusters NGC 1783, 1846, and 1978. Seven of the stars, which are carbon stars, are photometrically indistinguishable from carbon stars in the general field of the LMC. Bolometric magnitudes and temperatures are derived from the infrared data. The average bolometric magnitude of the carbon stars is -4.9 , which is about two magnitudes fainter than previously published values, but still high enough to require that these stars are asymptotic giant branch stars. The most luminous M-type stars in these clusters are warmer and perhaps more luminous than the giant branches of metal-rich Galactic globular clusters, in agreement with Hodge's results based on $U B V$ photometry. The location of the M-type stars may present some problems for recent giant branch model calculations.

Subject headings: clusters: open — galaxies: Magellanic Clouds — stars: late-type stars: luminosities
\end{abstract}

\section{INTRODUCTION}

The giant branches of the red, populous LMC clusters NGC 1783, 1846, and 1978 differ in several respects from those of Galactic globulars (Gascoigne 1962; Hodge 1960a, $b$; Hesser, Hartwick, and Ugarte 1976). In all three clusters there are bright stars redder than any found in Galactic globulars; the giant branches of NGC 1783 and 1846 are brighter than those of Galactic halo clusters; and both NGC 1846 and 1978 have giant branch slopes which are considerably less steep than those of Galactic halo clusters. Suggestions advanced (by the above cited authors and, e.g., Feast and Lloyd Evans 1973) to account for these differences include differences in age and chemical composition. The possibility that these clusters could contain numerous carbon stars led Mould and Aaronson (1979) to make a spectroscopic survey of giant branch tip stars in nine Large Magellanic Cloud (LMC) and Small Magellanic Cloud (SMC) clusters. They identified many stars, including several in NGC 1783,1846 , and 1978, as carbon stars and derived age estimates for the clusters based on estimated bolometric luminosities for the carbon stars.

In this paper we present near-infrared observations of some of the reddest stars in NGC 1783,1846, and 1978, including 11 of the stars observed by Mould and

${ }^{1}$ Cerro Tololo Inter-American Observatory is supported by the National Science Foundation under contract No. AST 78-27879.
Aaronson (1979, hereafter MA). These infrared data have the great advantage of giving bolometric luminosities and temperatures for cool stars directly since the measurements are in the spectral region of maximum stellar flux density. The importance of obtaining bolometric luminosities for these stars from infrared measurements has been emphasized by MA. Our luminosities and temperatures of the LMC stars are compared with those derived from infrared photometry of stars in Galactic globular clusters and with the recent theoretical giant branch tracks of Sweigart and Gross (1978).

\section{OBSERVATIONS}

The IR data presented in this paper were obtained with an InSb detector system and an f/30 chopping secondary on the CTIO $4 \mathrm{~m}$ telescope. This yields a scale of $1^{\prime \prime} .77 \mathrm{~mm}^{-1}$ in the focal plane-an important aid when working in fields as crowded as exist in these clusters. The filters and observing techniques employed are similar to those described in Frogel et al. (1978) and in Cohen, Frogel, and Persson (1978, hereafter CFP). An on-axis Quantex TV (looking through an IR/visual dichroic filter) was used for guiding. The area immediately around the star being measured and the two symmetrically placed sky positions were carefully inspected, and the throw of the chopping secondary was adjusted so as to place the sky 


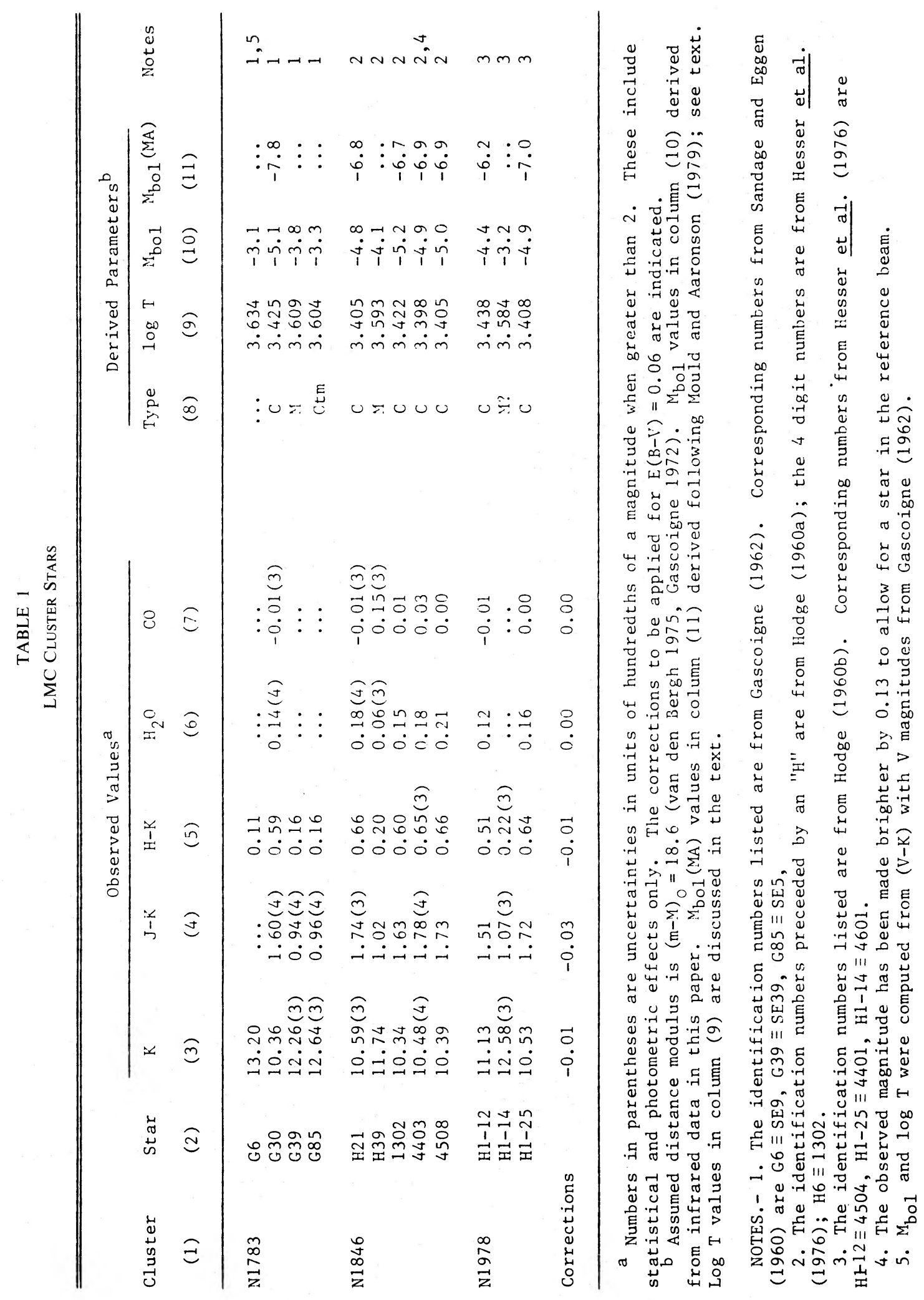




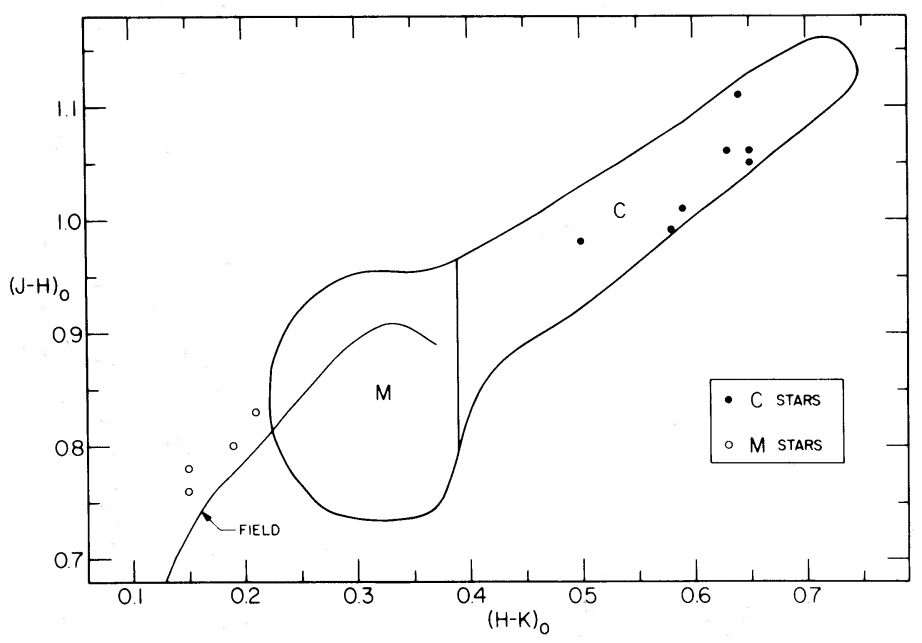

FIG. 1.- $(J-H)$ vs. $(H-K)$. The outlined areas encompass the locations of 70 late-type $\mathrm{C}$ and $\mathrm{M}$ stars observed in the field of the LMC and 32 Galactic $C$ stars as described in the text. The $C$ area contains only carbon stars, while the $M$ area predominantly contains $M$-type stars. The curved line is the mean relationship for Galactic K and $M$ giants. The open and filled circles are the LMC cluster stars from Table 1. Star NGC $1783-\mathrm{G} 85$ (type $\mathrm{Ctm}$ ) has been plotted as an M star on the basis of its $J H K$ colors.

positions away from any contaminating stars. In one case it was necessary to correct the magnitude for a star in one of the sky positions.

In NGC 1846 and 1978 the reddest uncrowded stars listed by Hodge $(1960 a, b)$ were observed. The work of Hesser, Hartwick, and Ugarte (1976) indicates the presence of several stars in NGC 1846 as red or redder than those selected from Hodge's $(1960 a, b)$ study, and these were also observed if possible. We included the reddest star found by Gascoigne (1962) in NGC 1783 and chose the other three in this cluster at random from the uncrowded stars having $B-V \gtrsim 1.6$. We also included stars observed by one of use (J. G. C.) in a separate spectroscopic program.

Columns (1) and (2) of Table 1 list the stars that were observed in the three LMC clusters. The IR magnitudes, colors, and absorption band indices, reduced to the system of standard stars of Frogel et al. (1978) and of Aaronson, Frogel, and Persson (1978), are given in columns (3)-(7). At the bottom of each column is the correction to be applied for an assumed reddening of $E(B-V)=0.06$ (van den Bergh 1975); the corrections are based on the van de Hulst curve number 15 (Johnson 1966).

\section{IDENTIFICATION OF C- AND M-TyPe STARS}

For all but one of the 12 stars in Table 1, MA have given rough spectroscopic classifications; these are listed in column (8). Comparison of the broad-band colors with the classifications show a clear separation between the $\mathrm{C}$ - and $\mathrm{M}$-type stars: the carbon stars are much redder in $J-K$ and $H-K$ color than are the $\mathrm{M}$ stars. This is shown in Figure 1, where we have plotted the data of Table 1 together with a schematic representation of the locations of 70 field $C$ and $M$ stars in the LMC. The boundaries of the regions enclose nearly all of the stars we have observed (data to be published) in unbiased samples from Blanco,
Blanco, and McCarthy's (1978, and private communication) spectroscopic survey of the "Bar West" and "Optical Center" LMC fields and of carbon stars with VRI photometry (Richer, Olander, and Westerlund 1979) from Westerlund et al.'s (1978) LMC survey. The region in Figure 1 labeled $\mathrm{C}$ is populated only by carbon stars, whereas the region labeled $\mathbf{M}$ is populated primarily by late $M$ giants. In addition, the members of a sample of 33 galactic carbon stars (data to be published) populate the same region as, and are indistinguishable from, the LMC C stars in Figure 1. Because of their IR colors and magnitudes, we will consider NGC 1783-G6 and NGC 1783-G85 as M stars.

Table 1 also shows that the LMC carbon stars have small $\mathrm{CO}$ indices, normally indicative of weak $\mathrm{CO}$ absorption. For carbon stars with $(J-K)>1.4$, the $\mathrm{CO}$ and $\mathrm{H}_{2} \mathrm{O}$ indices are strongly affected by the underlying stellar energy distribution in the $2.2 \mu \mathrm{m}$ window and not just by molecular absorption. The weakening of the $2.3 \mu \mathrm{m}$ CO bands can be clearly seen in high-resolution spectra of galactic carbon stars (Johnson and Méndez 1970; Frogel and Hyland 1972) and could be due to blanketing effects. These spectra also show that there is no $\mathrm{H}_{2} \mathrm{O}$ absorption in carbon stars; what our $\mathrm{H}_{2} \mathrm{O}$ index is in fact measuring is the slope of the continuum. The $\mathrm{CO}$ and $\mathrm{H}_{2} \mathrm{O}$ indices in Table 1 are completely consistent with those measured for LMC field and galactic carbon stars (data to be published).

The main point we wish to make from the above comparisons is that the IR energy distributions of the LMC field and cluster stars and C- and M-type stars in our galaxy are all quite similar. Thus we are confident that the bolometric corrections we shall derive on the basis of a comparison of near-IR data for LMC and Galactic stars will be unaffected by any systematic uncertainties. 


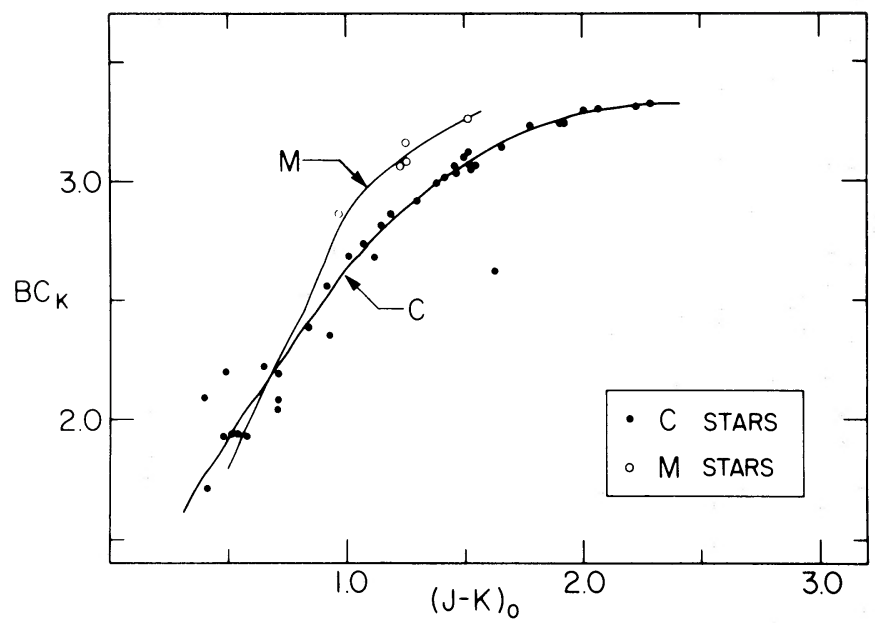

FIG. 2.-The bolometric corrections to the $K$-magnitudes as aa function of $(J-K)_{0}$ derived from observations of Galactic stars. The only star lying significantly off the adopted mean line is MJ number 9 which has a $(V-K)$ very blue for its $(J-K)$. For $(J-K)<0.95$ only the mean relation is shown as discussed in the text.

\section{BOLOMETRIC LUMINOSITIES AND TEMPERATURES}

For the stars observed in the present paper, we have computed bolometric luminosities and temperatures from the IR data alone. This is advantageous because of the possibility of variability and of crowding errors affecting the optical photometry. At longer wavelengths, the smaller amplitude of variability and the enhanced contrast between these very red stars and the bluer background act to minimize such errors.

The procedure we used to obtain the bolometric luminosities was first to find the bolometric corrections (BC) which apply to the $K$-magnitudes, i.e., $M_{\text {bol }}=K_{0}$ $+\mathrm{BC}_{K}$, where $\mathrm{BC}_{K}=(V-K)_{0}+\mathrm{BC}_{V}$, and $\mathrm{BC}_{V}$ is the ordinarily defined bolometric correction. To find the dependence of $\mathrm{BC}_{K}$ or $\mathrm{BC}_{V}$ on color, in this case $J-K$, we require complete energy distributions, but these are not available for the LMC stars. We therefore took the multicolor photometry of Mendoza and Johnson (1965, hereafter MJ) and integrated the energy distributions following the procedure described in Persson et al. (1980). It was necessary to transform MJ's $(J-K)$ colors to our system (Frogel et al. 1978) and to assume $(H-K)$ colors for the stars. These were derived from a mean $(J-K),(H-K)$ relation for our unpublished carbon star data. Our values of $\mathrm{BC}$ agree well with those of $\mathrm{MJ}$ (ours are systematically $0.07 \mathrm{mag}$ less at a given $[J-K])$. Figure 2 shows the tight dependence of $\mathrm{BC}_{K}$ on $(J-K)$ for all the carbon stars observed by $\mathrm{MJ}$. We have also indicated the mean relation between $\mathrm{BC}_{K}$ and $(J-K)$ for oxygen-rich giants based on the observations of $\mathrm{MJ}$ (points labeled $M$ in Fig. 2) and our own published data. ${ }^{2}$ Differences

${ }^{2}$ Values for $\mathrm{BC}_{V}$ for all the stars observed in CFP, Frogel, Persson, and Cohen (1979), and Persson et al. (1980) were recomputed as noted in Persson et al. (1980). The resulting dependence of $\mathrm{BC}_{V}$ on $(V-K)$ has a dispersion which is less than $\pm 0.05 \mathrm{mag}$ for a given $(V-K)$ and is independent of cluster metallicity as would be predicted from the models of CFP. The $\mathrm{BC}_{V},(V-K)$ dependence was combined with the mean $(V-K)$. $(J-K)$ relation of Frogel et al. (1978) to give the mean $\mathrm{BC}_{K},(J-K)$ relation in Figure 2 for oxygen-rich giants with $(J-K)<0.95$. between our BCs and those of MJ, Johnson (1966), and Lee (1970) for oxygen-rich stars are small, typically less than $0.1 \mathrm{mag}$.

Because there seem to be no significant differences in the energy distribution between the LMC cluster and field carbon stars and Galactic carbon stars, it is reasonable to use Figure 2 to obtain the bolometric magnitudes for the stars listed in Table 1. The values listed in column (10) are based on a distance modulus of $(m-M)_{0}=18.6$ for the LMC (van den Bergh 1975; Gascoigne 1972). We also computed values for $M_{\text {bol }}$ with bolometric corrections to the $K$-magnitude derived from $(V-K)_{0}$ by using the published $V$ magnitudes (Hodge 1960a, $b$; Gascoigne 1962) for nine of the $\mathrm{C}$ and $\mathrm{M}$ stars of Table 1 . The resulting absolute bolometric magnitudes were $0.10 \mathrm{mag}$ fainter in the mean with a dispersion of $0.11 \mathrm{mag}$. This good agreement arises at least in part because of the insensitivity of $\mathrm{BC}_{K}$ to color for stars whose energy distributions peak near $2 \mu \mathrm{m}$ (Dyck, Lockwood, and Capps 1974). Since our photometry is measuring a substantial fraction of the total luminosity of the carbon stars, the values of $M_{\text {bol }}$ in column (10) are unlikely to be in error by more than a few tenths, aside from uncertainties in the distance modulus of the LMC.

MA estimated values of $M_{\text {bol }}$ for these carbon stars from a set of optical colors and magnitudes given in their Table 1 and a relation between $\mathrm{BC}_{V}$ and color given in their Figure 3. Following the procedure outlined by MA, we arrived at the values tabulated in column (11) of our Table 1. These values are $2 \mathrm{mag}$ brighter in the mean than the values calculated on the basis of the new IR data. (Note that MA assumed zero reddening and 18.7 for the LMC distance modulus. Use of these values reduces the discrepancy by a small amount.) MA's procedure depends on a monochromatic $(15 \AA)$ color between $\lambda \lambda 5350$ and 6250 determined from fluxes for the LMC stars and a comparison of these colors with those determined from scans of Galactic C stars by Fäy et al. (1974). 


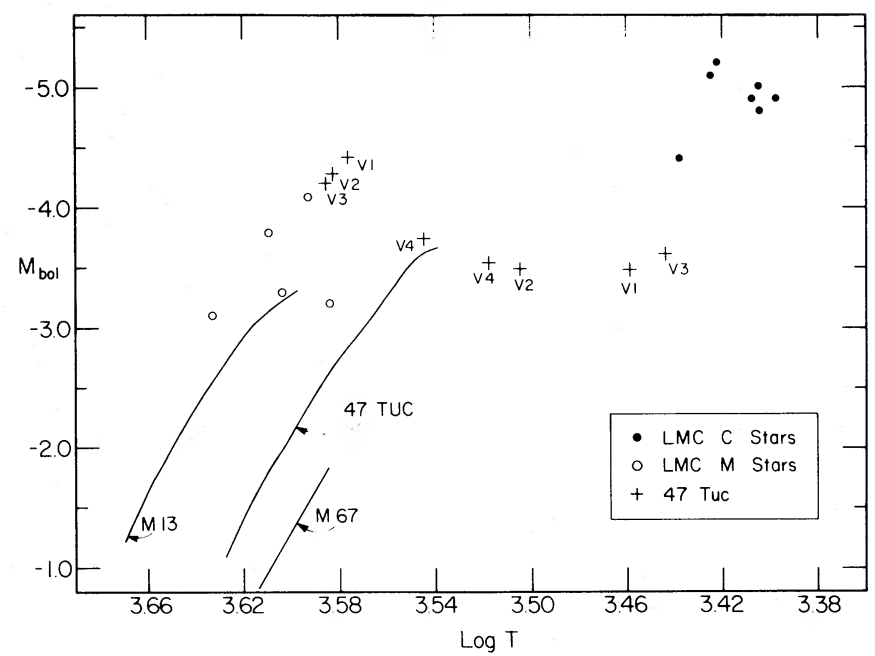

FIG. 3.-An H-R diagram for the LMC cluster stars, two Galactic globulars, and one open cluster, and for the 47 Tuc long-period variables as observed at maximum and minimum at $K$.

Inspection of the spectra illustrated by MA and by Fäy et al. (1974) indicates that such a color is severely influenced by line blanketing. It is not unreasonable to suppose that blanketing over small spectral intervals could be severely influenced by differences in the metallicities and/or mixing histories between the LMC and Galactic carbon stars. Such effects combined with the large slope of MA's $\mathbf{B C}_{V}$ versus color relation for colors less than 1.0 (their $\mathrm{BC}_{\nu} \mathrm{s}$ change by $5 \mathrm{mag}$ for a change in color of only 0.5 ) could easily introduce a large systematic error into the resulting values of $M_{\text {bol }}$. $\mathrm{MA}$ in fact noted that if they estimated bolometric corrections from a $(\mathrm{BC}, B-V)$ relation, the mean $M_{\text {bol }}$ for all of their LMC carbon stars would be $1 \frac{1}{2}$ mag fainter than that obtained by their method described above.

Our bolometric magnitudes are also on average fainter than those found for other LMC carbon stars by Crabtree, Richer, and Westerlund (1976) on the basis of $V-R$ colors and the $\mathrm{BC}_{V}, V-R$ relation of Olson and Richer (1975). However, the photometry of Crabtree, Richer, and Westerlund (1976) is superseded by that of Richer, Olander, and Westerlund (1979), and the $M_{\text {bol }}$ values of the latter authors are considerably fainter than those of Crabtree $e t$ al.

Effective temperatures for the LMC cluster M stars (col. [9], Table 1) were computed from their $(J-K)_{0}$ colors and the model atmospheres presented in CFP for stars of moderate metal deficiency. We estimate that the relative uncertainties in these temperatures are less than $\pm 150 \mathrm{~K}$ or $\pm 0.015 \mathrm{dex}$. As a check we also computed the $\mathrm{M}$ star temperatures from the $(V-K)_{0}$ colors. In the mean, these temperatures were only $55 \mathrm{~K}$ less than those computed from $(J-K)_{0}$.

Extensive discussions of the problems inherent in assigning effective temperatures to carbon stars on the basis of broad-band photometric observations have been given by Wallerstein (1973) and Scalo (1976) and will not be repeated here. The results of MJ show a reasonably good correlation between their derived temperatures and $(J-K)$ color. We used this correlation to obtain temperature estimates for the carbon stars of Table 1, and the values are given in column (9). Such temperatures, as Wallerstein (1973) has emphasized, should be regarded only as color temperatures. We estimate that these values could differ by more than $\pm 300 \mathrm{~K}(0.05 \mathrm{dex})$ from the effective temperatures. Until such temperatures can be better estimated, it is difficult to interpret the large gap between the clusters M's and C's in Figure 3.

\section{v. DISCUSSION}

The LMC cluster giants from Table 1 are shown on a luminosity-temperature plot in Figure 3. Fiducial giant branches for the Galactic globulars M13 and 47 Tucanae and for the old open cluster M67 are also indicated. ${ }^{3}$ The four reddest variables in 47 Tuc have been observed by us (Frogel, Persson, and Cohen $1980 \mathrm{~b}$, in preparation) over more than half of their periods, and their locations at maximum and minimum observed $K$-magnitudes are indicated in Figure 3 as well. ${ }^{4}$

\footnotetext{
${ }^{3}$ The data for M13 are from CFP; for M67 they are from CFP and from new observations of bright red giants which are cluster proper motion members (Frogel, Persson, and Cohen 1980a, in preparation); for $47 \mathrm{Tuc}$, the infrared data are from Frogel et al. (in preparation), while the optical data needed were obtained from Lee (1977). Lee gives $E_{B-V}=0.04$ and an observed $V$-magnitude for the horizontal branch of 47 Tuc of $14.06 \pm 0.12$. We assumed that $M_{V_{0}}$ for the HB was +0.8 and thus derive $(m-M)_{0}=13.14$ for 47 Tuc. For stars cooler than $4000 \mathrm{~K}$ we have used the revised temperature scales of Tsuji (1978) and Ridgeway et al. (1980).

${ }^{4}$ The bolometric corrections and effective temperatures for these 4 stars were calculated from their $(J-K)$ and $(V-K)$ colors at maximum and minimum light using techniques similar to those described above. For stars as red as these, $(V-K)$ varies typically between 4.0 and 8.5 , and yet the values of $\mathrm{BC}_{K}$ are not particularly sensitive to $(V-K)$. An uncertainty in $(V-K)$ of \pm 1.0 results in an uncertainty in $\mathrm{BC}_{K}$ of only \pm 0.2 .
} 


\section{a) The Carbon Stars}

Our new $M_{\text {bol }}$ values place the carbon stars in Figure 3 at $0.8-1.6$ mag brighter than the tip of the giant branch of 47 Tuc, which is still consistent with their being asymptotic giant branch (AGB) stars (e.g., Gingold 1974; Iben and Truran 1978) as was concluded by MA. However, they are not more than 0.6 mag brighter than the 47 Tuc variables at maximum light. These Mira variables may be AGB stars as well (Wood 1974). The significantly lower values of $M_{\text {bol }}$ as compared to the results of MA, though, removes the inconsistency between the luminosity predicted by MA for a cluster age of $3 \times 10^{9} \mathrm{yr}$ and a reasonable mass-loss rate, and their observed mean value for the carbon star luminosities. Furthermore, if we follow along with the scheme used by MA to predict AGB parameters, it is not necessary to invoke low helium abundances or very low (e.g., $1 \times 10^{9} \mathrm{yr}$ ) ages to account for the $M_{\text {bol }}$ of the carbon stars. We note, however, that Iben's (1975) mechanism for producing carbon stars via thermal pulsing which was called upon by MA has been found to have severe theoretical difficulties for stars with a mass low enough to be consistent with an age of $3 \times 10^{9}$ yr (Iben and Truran 1978).

\section{b) The M-Type Stars}

In Figure 3 the LMC cluster M-type stars have luminosities that scatter $( \pm 0.5 \mathrm{mag})$ around the giant branch tip luminosity of 47 Tuc. These luminosities are marginally consistent with expectations based on theoretical calculations for GB tip luminosities (e.g., Sweigart and Gross 1978 and Rood 1972 predict $M_{\text {bol }} \approx-3.6$ to -3.8 for moderately metal-deficient stars of approximately solar mass), and they are generally fainter than the 47 Tuc variables V1, V2, and V3 at maximum light.

The effective temperatures of the LMC M-type stars are somewhat higher than the values for the 47 Tuc GB stars of comparable luminosity and place the LMC stars in the vicinity of the tip of the M13 GB. However, there are at least two pieces of evidence which argue against the LMC stars being as metal poor as M13. First, the $\mathrm{CO}$ strength of the one star for which this index was observed (NGC 1846-H39) is comparable to that seen in the metal-rich globular cluster M71 (Frogel, Persson, and Cohen 1979). Second, the presence of $\mathrm{TiO}$ absorption (MA) in stars as hot as these implies that they cannot be as metal poor as stars in M13 (Mould, Stutman, and McElroy 1979, and references therein).

It has been suggested previously that the three clusters studied here are of intermediate age; the $\mathrm{M}$ type stars plotted in Figure 3 provide us with a quantitative test of this hypothesis. At $M_{\text {bol }}=-3.0$, the difference in $\log T_{e}$ between the LMC giants and the 47 Tuc giant branch is about 0.06 . From the giant branch tracks of Sweigart and Gross (1978), we find that irrespective of metallicity, the maximum $\Delta \log T_{e}$ that can be produced at this luminosity by varying the mass of the red giants (hence the age of the cluster) is only $0.04 \mathrm{dex}$. For the sequence of models with $Y$ $=0.30$ and $Z=0.01$, this corresponds to a range in red giant mass from $0.8 M_{\odot}$ (that presumably characteristic of $47 \mathrm{Tuc}$ ) to $1.8 \mathrm{M}_{\odot}$, and hence to an age difference of $14 \times 10^{9} \mathrm{yr}$ according to the Ciardullo and Demarque (1977) isochrons. One cannot go to higher masses and thus lower ages to increase $\Delta \log T_{e}$, as at $\sim 2 M_{\odot}$ helium begins to burn quietly and the evolutionary tracks for the GB do not reach high enough luminosities (Sweigart and Gross 1978). We note that the Sweigart and Gross (1978) models are consistent with the locations of the 47 Tuc and M67 giant branches if allowance is made for small differences in age and chemical compositions between these two clusters.

An increase in the helium abundance of the LMC will act to increase the temperature of the giant branch. However, an increase in $Y$ to 0.40 increases $\log T_{e}$ at constant age and at a fixed luminosity by less than 0.01 dex. Although the giant branch location is most sensitive to metallicity, we hesitate to ascribe as much as $0.04 \mathrm{dex}$ in $\log T_{e}$ to this factor as we have already given reasons for doubting such a low metallicity $([\mathrm{Fe} / \mathrm{H}] \approx-1.8)$ for these LMC clusters.

The observed difference of $0.06 \mathrm{dex}$ in $\log T_{e}$ between the tip of the giant branches of 47 Tuc and the LMC clusters is thus marginally inconsistent with model predictions. Consistency would require a very young age together with a metal abundance lower than that of 47 Tuc. As noted by MA, ages less than 1 $\times 10^{9}$ yr can be ruled out by comparing the integrated colors of these clusters (van den Bergh and Hagen 1968) corrected for reddening, with the predictions for the $U B V$ colors of evolving clusters by Searle, Sargent, and Bagnuolo (1973). Perhaps an easy way out of this problem is to question some of the assumptions of the model calculations. For example, could there be a systematic dependence of the ratio of mixing length to pressure scale height on stellar mass (or equivalently cluster age)? The calculations reviewed by Sweigart (1978) indicate that for values of this ratio between 0.5 and 2.0 a doubling of the value increases $\log T_{\text {eff }}$ at the GB tip by 0.07 . Simoda and Iben (1970) have suggested that it may be best to use observations to determine the values of theoretical parameters such as the mixing length-to-scale height ratio.

\section{SUMMARY}

The results of this paper can be summarized as follows:

1. The near-IR energy distributions of 12 LMC cluster late-type stars are similar to those of field LMC stars and to those in our Galaxy.

2. Bolometric corrections which are relatively free of uncertainty have been derived from the IR photometry. For a true distance modulus of 18.6 for the LMC, the mean absolute magnitude of seven cluster carbon stars is $M_{\text {bol }}=-4.9$, only a few tenths more luminous than the 47 Tuc variables at maximum light, 
but sufficiently above the theoretical red giant tip that the LMC cluster carbon stars are probably asymptotic giant branch stars. This value is considerably fainter than some previous estimates, but is consistent with MA's conclusion that these clusters are of intermediate age.

3. The LMC cluster M stars lie close to the tip of the M13 giant branch in the $M_{\text {bol }}-\log T_{e}$ plane, yet from independent evidence we believe that the LMC clusters have metallicities characteristic of the more metal-rich globulars. It appears somewhat difficult to theoretically account for the location of the $M$ stars by varying age, metallicity, and helium content of the LMC clusters within reasonable limits.

J. A. F. would like to thank J. Baldwin and J. Elias for assistance with the $4 \mathrm{~m}$ observations and several of his colleagues at CTIO for useful comments on a draft of this paper. We are grateful to J. Mould and A. Aaronson for discussing their data with us before publication and for some valuable criticism of the present paper. We appreciate the continuing interest of V. M. Blanco in this project and the use, before publication, of his finding charts and coordinates.

\section{REFERENCES}

Aaronson, M., Frogel, J. A., and Persson, S. E. 1978, Ap. J., 220, 98 Blanco, B. M., Blanco, V. M., and McCarthy, M. F. 1978, Nature, 271, 638.

Ciardullo, R. B., and Demarque, P. 1977, Trans. Astr. Obs. Yale University, Vol. 35.

Cohen, J. G., Frogel, J. A., and Persson, S. E. 1978, Ap. J., 222, 165 (CFP)

Crabtree, D. R., Richer, H. B., and Westerlund, B. E. 1976, Ap. J. (Letters), 203, L81.

Dyck, H. M., Lockwood, G. W., and Capps, R. W. 1974, Ap. J., 189, 89.

Fäy, T. D., Jr., Warren, W. H., Jr., Johnson, H. R., and Honeycutt, R. K. 1974, A.J., 79, 634.

Feast, M. W., and Lloyd Evans, T. 1973, M.N.R.A.S., 164, 15P

Frogel, J. A., and Hyland, A. R. 1972, Proc. 17th International Astrophysical Symposium, Liège, p. 111.

Frogel, J. A., Persson, S. E., Aaronson, M., and Matthews, K. 1978, Ap. J., $220,75$.

Frogel, J. A., Persson, S. E., and Cohen, J. G. 1979, Ap. J., 227, 499

Gascoigne, S. C. B. 1962, M.N.R.A.S., 124, 201. 1972, Quart. J.R.A.S., 13, 274.

Gingold, R. A. 1974, Ap. J., 193, 177.

Hesser, J. E., Hartwick, F. D. A., and Ugarte, P. 1976, Ap. J. Suppl., 32, 283.

Hodge, P. W. 1960a, Ap. J., 132, 341. $1960 b, A p . J ., 132,246$

Iben, I., Jr. 1975, Ap. J., 196, 525.

Iben, I., Jr., and Truran, J. W. 1978, Ap. J., 220, 980

Johnson, H. L. 1966, in Nebulae and Interstellar Matter, ed. B. M. Middlehurst and L. M. Aller (Chicago: University of Chicago Press), p. 167.
Johnson, H. L., and Méndez, M. E. 1970, A.J., 75, 785.

Lee, S.-W. 1977, Astr. Ap. Suppl., 27, 381.

Lee, T. A. 1970, Ap. J., 162, 217.

Mendoza, E. E., and Johnson, H. L. 1965, Ap. J., 141, 161 (MJ).

Mould, J., and Aaronson, M. 1979, Ap. J., 232, 421 (MA).

Mould, J., Stutman, D., and McElroy, D. 1979, Ap. J., 228, 423.

Olson, B. I., and Richer, H. B. 1975, Ap. J., 200, 88.

Persson, S. E., Frogel, J. A., Cohen, J. G., Aaronson, M., and Matthews, K. 1980, Ap. J., 235, 452.

Richer, H. B., Olander, N., and Westerlund, B. E. 1979, Ap. J., 230, 724.

Ridgway, S. T., Joyce, R. R., White, N. M., and Wing, R. F. 1980, Ap. J., 235, 126.

Rood, R. T. 1972, Ap. J., 177, 681.

Sandage, A. R., and Eggen, O. J. 1960, M.N.R.A.S., 121, 232.

Scalo, J. M. 1976, Ap. J., 206, 474.

Searle, L., Sargent, W. L. W., and Bagnuolo, W. G. 1973, Ap. J., 179, 427.

Simoda, M., and Iben, I., Jr. 1970, Ap. J. Suppl., 22, 81.

Sweigart, A. V. 1978, in IAU Symposium 80, The HR Diagram, ed. A. G. D. Philip and D. S. Hayes (Dordrecht: Reidel), p. 333.

Sweigart, A. V., and Gross, P. G. 1978, Ap. J. Suppl., 36, 405.

Tsuji, T. 1978, Astr. Ap., 62, 29.

van den Bergh, S. 1975, in Galaxies and the Universe, ed. A. Sandage, M. Sandage, and J. Kristian (Chicago: University of Chicago Press), p. 509

van den Bergh, S., and Hagen, G. L. 1968, A.J., 73, 569.

Wallerstein, G. 1973, Ann. Rev. Astr. Ap., 11, 115.

Westerlund, B. E., Olander, N., Richer, H. B., and Crabtree, D. R. 1978, Astr. Ap. Suppl., 31, 61.

Wood, P. R. 1974, Ap. J., 190, 609.

Note added in proof. - For four of the five M stars in Table 1, the temperatures based on $(J-K)_{0}$ colors were not converted to the Ridgway et al. (1980) scale (see n. 3). The exact relationship between the observed $J-K$ colors and $V-K$, on which the Ridgway et al. (1980) scale is based, depends on metallicity for stars as cool as these. In particular we expect that there will be differential blanketing effects due to TiO in the $V$-filter and $\mathrm{H}_{2} \mathrm{O}$ in the $K$-filter. Effects of the latter molecule are evident, for example, in the spectra of Johnson and Méndez (1970). Because of the reduced sensitivity of $J-K$ to $T_{\text {eff }}$ as compared to $V-K$, knowledge of the exact variation of molecular line absorption with abundance becomes critical. The $J-K$ and $V-K$ colors could easily be affected at the 0.05 and at the $0.20 \mathrm{mag}$ level, respectively. We note, though, that at least for $V-K$ bluer than 3.9 , the Ridgway et al. (1980) and CFP temperature scales agree to within $100 \mathrm{~K}$, independent of metallicity, even though the CFP models did not include the effects of molecular absorption. Thus for temperatures greater than $3800 \mathrm{~K}$ the effects of molecular absorption would appear not to significantly affect the determination of $T_{\text {eff }}$ from $V-K$. Of course, this may in part be due to the great sensitivity of $V-K$ to $T_{\text {eff }}$-a change in $T_{\text {eff }}$ from 3800 to $4000 \mathrm{~K}$ implies a change in $V-K$ of nearly $0.5 \mathrm{mag}$. The temperatures derived from the $(V-K)_{0}$ colors of the $\mathrm{M}$ stars in Table 1 are $\log T_{\text {eff }}=3.634,3.593,3.594,3.585$, and 3.611 for N1783-G6, -G39, -G85, N1846-H39, and N1978 H1-14, respectively, close to those given in Table 1 as described in $\S$ IV. These temperatures do not remove the discrepancy between the expected and empirical effective temperatures that is discussed in $\S \mathrm{V} b$.

J. G. Cohen: Division of Physics and Astronomy, California Institute of Technology, Pasadena, CA 91125

JAY A. Frogel: Cerro Tololo Inter-American Observatory, Casilla 603, La Serena, Chile

S. E. Persson: Hale Observatories, Carnegie Institute of Washington, Pasadena, CA 91101 